\title{
Identification of dimeric and oligomeric complexes of the human oxytocin receptor by co-immunoprecipitation and bioluminescence resonance energy transfer
}

\author{
D Devost and H H Zingg \\ Laboratory of Molecular Endocrinology, McGill University Health Center, Montreal, Quebec, Canada H3A 1A1 \\ (Requests for offprints should be addressed to H H Zingg, Laboratory of Molecular Endocrinology, Royal Victoria Hospital, 687 Pine Avenue West, \\ Montreal, Quebec, Canada H3A 1A1; Email: hans.zingg@mcgill.ca)
}

\begin{abstract}
The nonapeptide hormone oxytocin exerts many important biological functions, including uterine contractions during parturition and milk ejection during lactation. The manifold effects of oxytocin are mediated by a single oxytocin receptor (OTR) type, a member of the super-family of G-protein-coupled receptors. There is accumulating recent evidence that certain G-protein-coupled receptors exist in the form of oligomeric complexes. Here we demonstrate, using two different co-immunoprecipitation strategies as well as bioluminescence resonance energy transfer techniques, that the OTR is capable of forming oligomeric complexes in vivo and that these complexes exist at the cell surface membrane. The human OTR was $\mathrm{N}$-terminally tagged with either a Myc or Flag epitope and transiently expressed in COS-7 cells. Cell lysates were immunoprecipitated using an anti-Flag antibody and analyzed by SDS-PAGE and Western blotting using an anti-Myc antibody, or vice versa. Either strategy provided evidence for the co-precipitation of Myc- or Flag-tagged OTR respectively.

Biochemical characterization of OTR dimers showed that homodimer formation is not dependent on the establishment of disulfide bonds. The existence of OTR dimers and oligomers at the level of the cell surface was demonstrated by exposing intact living cells to an anti-Flag antibody and analyzing the immunoprecipitate by Western blotting with an anti-Myc antibody. This approach demonstrated furthermore that the presence of receptor oligomers at the cell surface is modulated by ligand in a time-dependent fashion. Finally, we obtained evidence that the OTR is forming oligomeric structures in intact living cells by observing the occurrence of bioluminescence resonance energy transfer in cells co-transfected with OTR constructs bearing at their C-terminus either a Renilla luciferase or the yellow fluorescent protein. Taken together, these data show that the OTR can form homodimers and oligomers in the cell model used and that these oligomers are present at the cell surface.
\end{abstract}

Journal of Molecular Endocrinology (2003) 31, 461-471

\section{Introduction}

The receptor for the multifunctional cyclic nonapeptide oxytocin (OT) is a G-protein-coupled receptor (GPGR) that forms part of the OT/ vasopressin receptor family. The OT receptor (OTR) is able to couple functionally to both $\mathrm{Gq} / 11$ and Gi proteins (Ku et al. 1995, Strakova \& Soloff 1997). OTR activation triggers multiple molecular events, including an increase in intracellular free calcium concentration, activation of mitogenactivated protein kinase, protein kinase- $\mathrm{C}$ and myosin light-chain kinase and synthesis of prostaglandin E2 (Zingg \& Laporte 2003). The mechanisms by which the multiple OTR signaling cascades are specifically activated and modulated are still incompletely understood. Several studies have assessed the ligand/receptor interactions between the OTR and OT by photoaffinity labeling (Breton et al. 2001), site-directed 
mutagenesis (Fanelli et al. 1999, Hawtin et al. 2001) or the construction of receptor chimeras (Postina et al. 1996).

All these studies refer to the OTR in its monomeric form. However, a growing body of evidence now indicates that certain GPCRs function as oligomeric complexes (Angers et al. 2002, Eidne 2002). Complexes can form between the same or different classes of receptors and the oligomerization process can be either dependent on (Patel et al. 2002) or independent of ligand binding (Issafras et al. 2002). Furthermore, many reports show the importance of GPCR oligomerization for multiple aspects of receptor biology such as intracellular signaling, trafficking, pharmacology and sequestration (Salahpour et al. 2000, Angers et al. 2002).

A complete understanding of OTR structure/ function relationships requires a definition of the oligomeric state of the OTR in vivo. As a first step towards resolving this problem, we have applied different co-immunoprecipitation strategies as well as an in vivo approach. We now report that the human OTR can form dimers and oligomers as demonstrated in vitro by co-immunoprecipitation and in vivo by bioluminescence resonance energy transfer (BRET) in transiently transfected COS-7 cells. The characterization of such complexes will be a prerequisite for a full understanding of the mechanism of OTR action.

While the present paper was under review, the formation of OTR dimers during biosynthesis was described by S. Terrillon et al. (2003).

\section{Materials and methods}

\section{DNA plasmids}

Plasmids encoding the wild-type human OTR or recombinant OTRs tagged at the N-terminus with either a Myc or a Flag epitope were a generous gift of Dr Bice Chini, Milan, Italy (Flag-OTR and Myc-OTR respectively). The plasmid B2R-YFP, encoding the human bradykinin $\mathrm{B} 2$ receptor with the coding region for the yellow fluorescent protein (YFP) added in frame at its C-terminus, was a gift from Dr S A Laporte, Montreal, Canada. The following additional constructs were derived: $3 \times$ Flag-OTR, an OTR construct with a triple Flag epitope added to the N-terminus by inserting the OTR coding sequence in frame into the expression vector p3 $\times$ Flag-CMV-7·1 (Sigma); OTR-luc, an OTR construct with the coding sequence for Renilla luciferase added in frame to the OTR C-terminus by inserting the OTR coding region into the vector pRL-CMV (Promega); OTR-YFP, an OTR construct with the coding sequence for YFP added in frame to the OTR C-terminus, obtained by inserting the OTR coding region in frame into the vector pEYFP (Clontech, Palo Alto, CA, USA). The sequence integrity of all constructs was verified by dideoxy sequencing.

\section{Tissue culture, transfections and co-immunoprecipitations}

COS-7 cells were cultured in DMEM 'high glucose' media (Gibco) supplemented with 10\% fetal bovine serum. The cells were cultured in the absence of antibiotics to ensure contamination-free conditions. The day before transfection, COS-7 cells were plated at a density of $2 \times 10^{6}$ cells per $10 \mathrm{~cm}$ dish. Cells were transiently transfected with a total of $5 \mu \mathrm{g}$ DNA using Effectene (Qiagen) according to the manufacturer's recommendations. Cell medium was changed the following day. Forty-eight hours post-transfection, cells were lysed at room temperature for $30 \mathrm{~min}$ in $1 \mathrm{ml}$ lysate buffer (25 mM Hepes (pH 7•4), $5 \mathrm{mM}$ EDTA, $50 \mathrm{mM} \mathrm{NaCl}, 10 \%$ glycerol, $1 \%$ Triton X-100, $50 \mathrm{mM}$ NaF, $30 \mathrm{mM}$ sodium pyrophosphate and protease inhibitors $(1 \mu \mathrm{g} / \mathrm{ml}$ each of aprotinin, leupeptin and pepstatin $\mathrm{A}$, and $1 \mathrm{mM}$ phenylmethylsulfonyl fluoride)). To avoid any non-specific OTR aggregation caused by disulfide bond formation, the sulfhydryl alkylating reagent iodoacetamide (IOA) was present at a concentration of $10 \mathrm{mM}$ during the solubilization and immunoprecipitation steps. The following steps were done at $4{ }^{\circ} \mathrm{C}$, except where stated otherwise. The lysates were cleared by centrifugation at $13400 \boldsymbol{g}$ for $10 \mathrm{~min}$ and protein content of the supernatant was evaluated using a BCA Protein Assay Reagent Kit (Pierce, Brockville, Ontario, Canada). For coimmunoprecipitation, 500-1000 $\mathrm{g}$ protein/ml were mixed with either $10 \mu \mathrm{g}$ anti-Myc antibody (clone 9E10; Upstate Biotechnology, Lake Placid, NY, USA) or $4 \mu \mathrm{g}$ anti-Flag antibody (Sigma) for $3 \mathrm{~h}$. The antibody-antigen complexes were precipitated by protein G-linked Sepharose (Pharmacia) for $30 \mathrm{~min}$ and the beads were washed twice with lysate buffer (without IOA). The beads were 
resuspended for $15 \mathrm{~min}$ at room temperature in $20 \mu \mathrm{l}$ urea buffer $(9.6 \mathrm{M}$ urea, $4 \%$ SDS, $250 \mathrm{mM}$ Tris (pH 6.8), 10\% glycerol, $130 \mathrm{mM}$ dithiothreitol (DTT) and bromophenol blue). In some experiments, to avoid non-reduced immunoglobulins masking high molecular complexes, the coimmunoprecipitation was performed using the anti-Flag antibody covalently linked to protein $\mathrm{G}$ Sepharose according to the protocol of Schneider et al. (1982). The denatured coimmunoprecipitation products were subjected to SDS-PAGE analysis and then electro-blotted onto PVDF membranes (Immobilon-P; Millipore, Bedford, MA, USA) overnight at $45 \mathrm{~V}$ in $10 \mathrm{mM}$ 3-(cyclohexylamino)-1-propane-sulfonic acid buffer (pH 11.0) containing 10\% methanol. The tagged receptors were immunodetected using the same antibodies used previously for coimmunoprecipitation in conjunction with a secondary anti-mouse antibody conjugated to horseradish peroxidase C. Bands were visualized using a chemiluminescent detection system (SuperSignal; Pierce).

\section{Cell surface co-immunoprecipitation}

Forty-eight hours post-transfection, COS-7 cells were stimulated or not by $100 \mathrm{nM}$ OT for different times at $37^{\circ} \mathrm{C}$. At the end of the incubation period, the cells were washed twice with ice-cold PBS, followed by one wash in Krebs/Hepes buffer (146 mM NaCl, 4.2 mM KCl, 0.5 mM $\mathrm{MgCl}_{2}$, $10 \mathrm{mM}$ Hepes $(\mathrm{pH} 7 \cdot 4)$ and $5.9 \mathrm{mM}$ glucose). Five milliliters of Krebs/Hepes containing $0.5 \%$ BSA and $10 \mu \mathrm{g} / \mathrm{ml}$ anti-Flag antibody were added to each dish and left overnight at $4{ }^{\circ} \mathrm{C}$. The plates were extensively washed with $\mathrm{PBS}$ and cells were lysed as before except that no IOA was added to the lysate buffer. Whole-cell lysates were then incubated with the protein G Sepharose and co-immunoprecipitation complexes were treated and analyzed as mentioned earlier.

\section{BRET}

The oligomerization of OTR in vivo was assessed by BRET in transiently transfected COS-7 cells. Twenty-four hours after transfection, cells were replated onto a 96-well plate at $10^{5}$ cells/well. The following day, medium was replaced with $100 \mu \mathrm{l}$ Krebs/Hepes buffer following three washes with the same buffer. Coelenterazine $h$ (Molecular Probes, Eugene, OR, USA) was added at a final concentration of $10 \mu \mathrm{M}$, and luminescence emission was measured immediately thereafter at both $460 \mathrm{~nm}$ and $530 \mathrm{~nm}$ using an FL 600 spectrofluorometer (Bio-Tek Instruments Inc., Winooski, VT, USA). The BRET ratio was calculated using the formula: (luminescence ${ }_{530 \mathrm{~nm}}-$

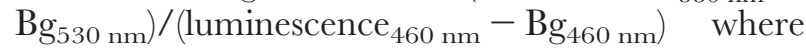
$\mathrm{Bg}_{\mathrm{x}}$ is the background at wavelength $\mathrm{x} \mathrm{nm}$, obtained by taking a luminescence measurement in the absence of substrate.

\section{Results}

\section{Evidence for OTR oligomerization by co-immunoprecipitation}

To investigate whether OTR can form dimers and oligomers, we first developed a coimmunoprecipitation strategy using two different OTR constructs that carried at their N-terminus either the Flag (Flag-OTR) or the Myc epitope (Myc-OTR). Co-immunoprecipitation of Flag immunoreactivity with the anti-Myc antibody or co-immunoprecipitation of Myc immunoreactivity with the anti-Flag antibody was taken as evidence for the existence of a physical association between Flag-OTR and Myc-OTR. The results of coimmunoprecipitation experiments using either of these approaches are shown in Fig. 1. Mycimmunoreactive bands were detected by Western blotting of Flag immunoprecipitates from cells transfected with both Flag-OTR and Myc-OTR plasmids (Fig. 1A, lane 1), but not from cells transfected with Myc-OTR alone (lane 2). Conversely, Flag-immunoreactive bands were detected by Western blotting of Myc immunoprecipitates from cells transfected with both Flag-OTR and Myc-OTR plasmids (Fig. 1B, lane 4), but not from cells transfected with Flag-OTR alone (lane 5). The bands detected corresponded to the molecular mass of the glycosylated form of the OTR at $70-80 \mathrm{kDa}$ (Breton et al. 2001), the non-glycosylated form of the OTR at $38 \mathrm{kDa}$ (Breton et al. 2001) and higher molecular mass complexes that correspond most likely to SDSresistant dimers and oligomers.

Receptor density in this in vitro system was $635 \pm 47 \mathrm{fmol} / \mathrm{mg}$ protein $(n=3)$, as determined by Scatchard analysis. Because, in vivo, OTR densities 

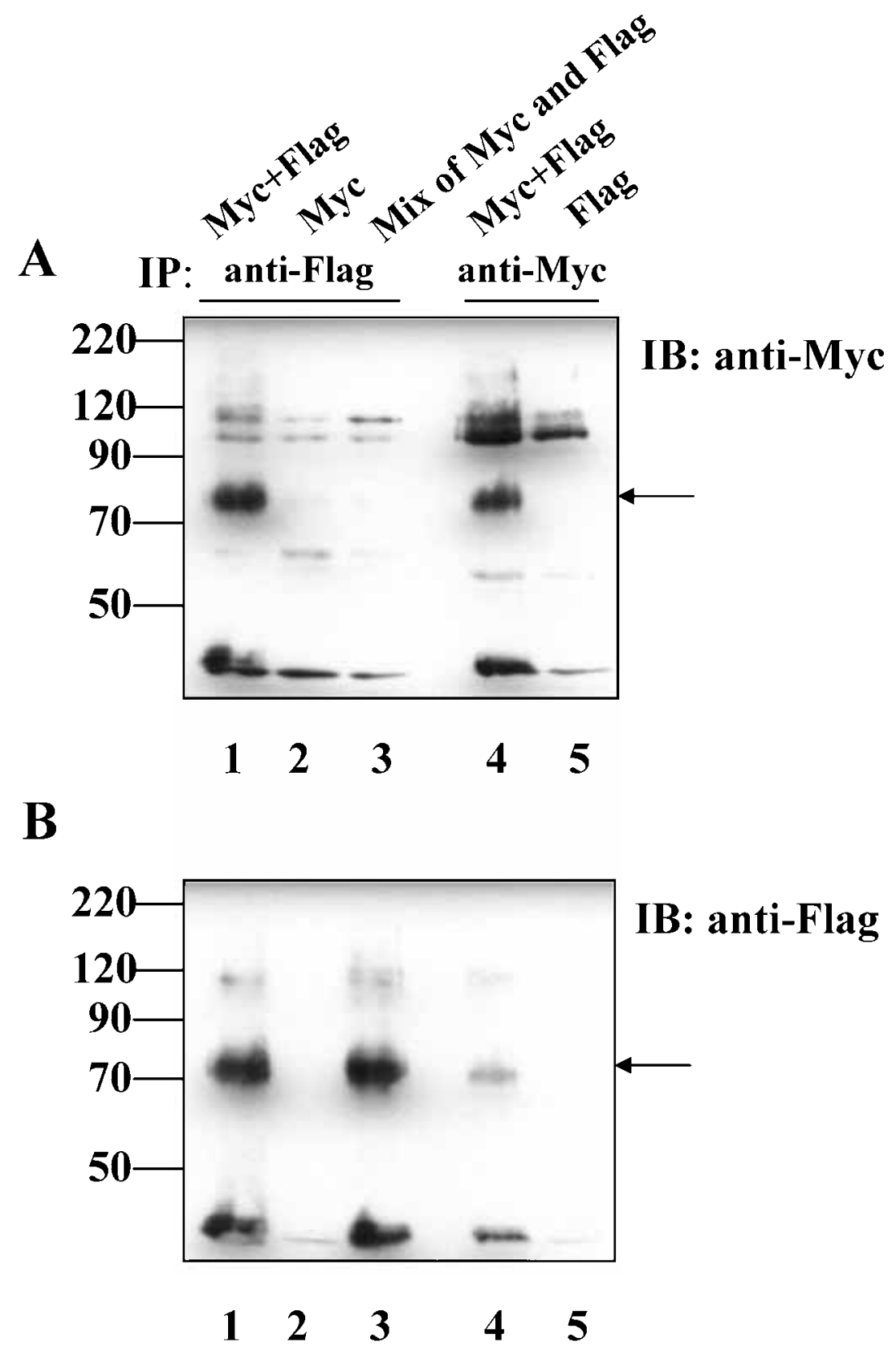

Figure 1 Demonstration of human OTR oligomerization by co-immunoprecipitation. (A) Western blot analysis of immunoprecipitates of total lysates of COS-7 cells transiently transfected with expression vectors encoding N-terminally tagged OTR. Cells were transfected as follows: lanes 1 and 4: Myc-OTR and Flag-OTR; lane 2: Myc-OTR alone; lane 3: mixture of two cell populations transfected separately with Myc-OTR or Flag-OTR; lane 5: Flag-OTR alone. Immunoprecipitation was performed using either an anti-Flag antibody (lanes 1-3) or an anti-Myc antibody (lanes 4 and 5) and immunodetection occurred with an anti-Myc antibody. (B) Same blot as in (A) following stripping and immunoblotting with an anti-Flag antibody. Arrows indicate bands corresponding in molecular mass to the glycosylated form of the OTR. Molecular mass markers (on the left) are in $\mathrm{kDa}$. The results presented are representative of two independent series of experiments. IB, immunoblot; IP, immunoprecipitate. 


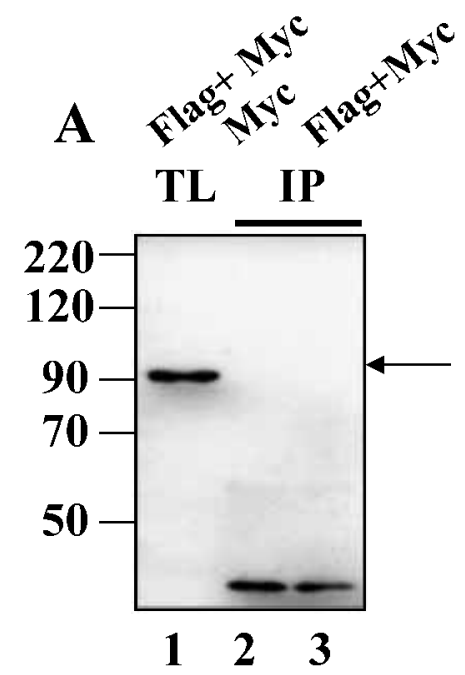

IB: anti-Na/K-ATPase
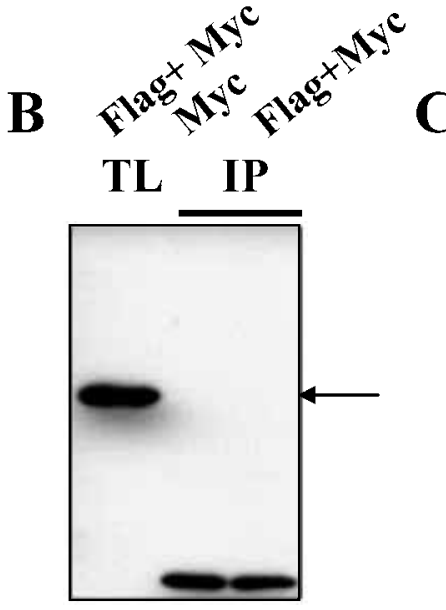

$\begin{array}{lll}1 & 2 & 3\end{array}$

anti-BiP

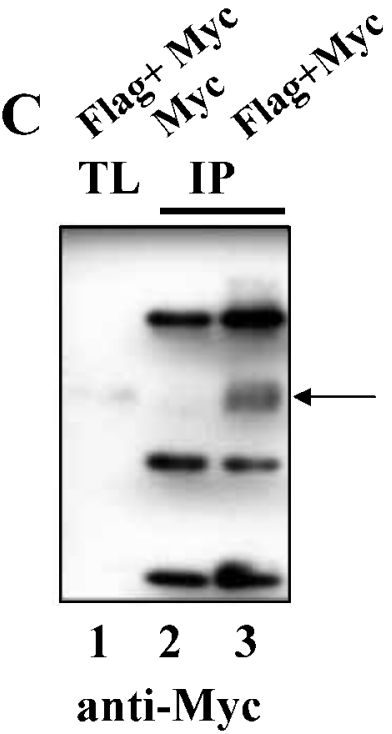

Figure 2 Absence of co-immunoprecipitation of abundant markers for the plasma membrane (Na/K-ATPase) or for the endoplasmic reticulum (BiP/GRP74). COS-7 cells were transfected with expression vectors for Flag-OTR and Myc-OTR (lanes 1 and 3) or Myc-OTR alone (lane 2). Total lysates (lane 1) or Flag immunoprecipitates (lanes 2 and 3) were analyzed by immunoblotting. The same membrane was immunoblotted sequentially with an anti-Na/K-ATPase antibody (Upstate Biotechnology) (A), an anti-Bip antibody (BD Biosciences, Oakville, Ontario, Canada) (B) or, as a positive control, with an anti-Myc antibody $(C)$. Specific bands are indicated by arrows. Molecular mass markers (on the left) are in $\mathrm{kDa}$. The results presented are representative of two independent series of experiments. IB, immunoblot; IP, immunoprecipitate; TL, total lysate.

can reach up to 1850 and $7300 \mathrm{fmol} / \mathrm{mg}$ protein in myometrium and endometrium respectively (Fuchs et al. 1992), these levels were well within the physiological range. In addition, when OTR expression levels were lowered by lowering the amount of expression plasmid used for transfection, OTR oligomers remained detectable by the co-immunoprecipitation approach at receptor densities as low as $50 \mathrm{fmol} / \mathrm{mg}$ protein.

Additional experiments were undertaken to assess the specificity of these observations. To exclude the possibility that the observed oligomerization of OTR could be due to artifactual oligomerization occurring after solubilization, we mixed, prior to immunoprecipitation, lysates from two cell populations, each expressing one of the two tagged receptor types. No specific bands were detected in this case (Fig. 1A, lane 3). To test whether the observed co-immunoprecipitation could be due to incomplete membrane solubilization or non-specific protein aggregation, we assessed whether abundant and ubiquitous proteins were co-immunoprecipitated. To this end, we determined whether $\mathrm{Na} / \mathrm{K}$-ATPase (a marker of the plasma membrane) or the chaperone protein BiP/GRP74 (a marker of the endoplasmic reticulum) was present in immunoprecipitates. Neither of these proteins was detected in immunoprecipitates (Fig. 2A and B), whereas OTR coimmunoprecipitation remained readily detectable (Fig. 2G).

\section{OTR oligomers are insensitive to reducing conditions}

There is no consensus in the literature whether GPCR oligomerization involves the formation of disulfide bonds. Whereas some GPCR oligomers have been reported to be sensitive to reducing conditions, others have been shown to be insensitive. To test the involvement of disulfide bonds in the formation of OTR oligomers, we compared the band pattern obtained by Western blotting of co-immunoprecipitation samples treated or not with the reducing agent DTT. In the absence of DTT treatment, a high molecular mass 


\section{IP:anti-Flag IB:anti-Myc}

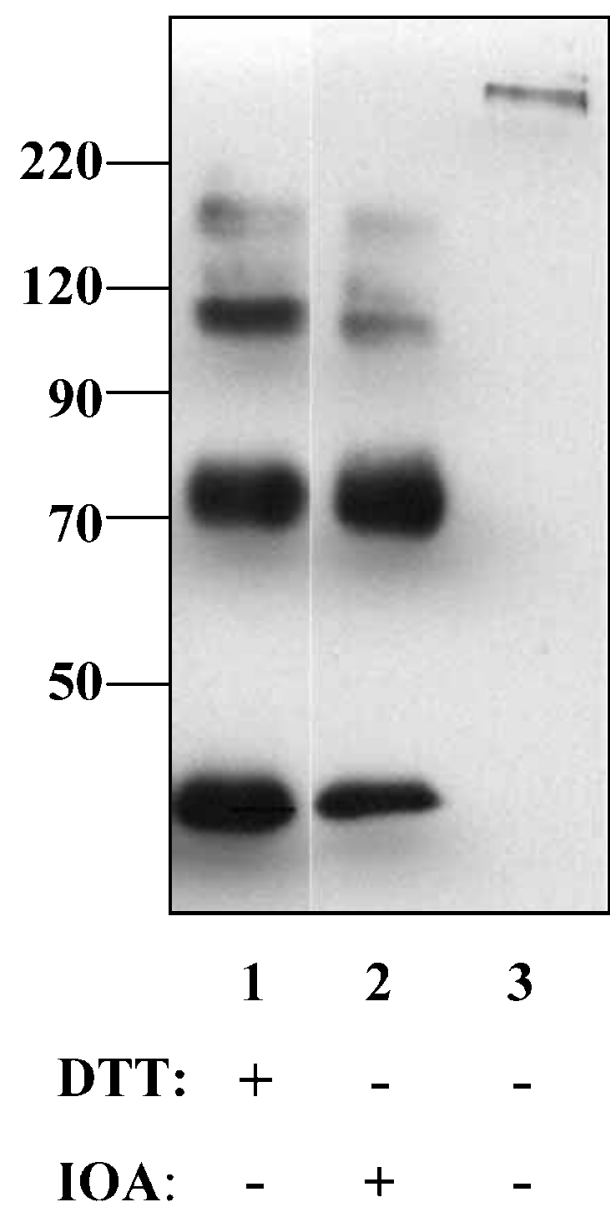

Figure 3 OTR oligomers are insensitive to reducing conditions. Lysates of COS-7 cells transiently transfected with Myc-OTR and Flag-OTR were immunoprecipitated using an anti-Flag antibody covalently linked to protein G Sepharose, in either the presence or absence of the sulfhydryl alkylating agent IOA (as indicated). The immunoprecipitates were denatured in either the presence or absence of the reducing agent DTT (as indicated) and immunoblotted using an anti-Myc antibody. Molecular mass markers (on the left) are in $\mathrm{kDa}$. The results presented here are representative of two independent series of experiments. IB, immunoblot; IP, immunoprecipitate.

aggregate was seen (Fig. 3, lane 3). If de novo formation of disulfide bonds in vitro during the extraction and precipitation steps was prevented by addition of IOA, only bands responding to receptor monomers, SDS-resistant dimers and some higherorder oligomers were detected (Fig. 3, lane 2). This indicated that the high molecular mass aggregates seen in lane 3 were due to disulfide bond formation that had occurred during the solubilization and precipitation steps in the non-reducing in vitro environment. As shown in lane 1, addition of the reducing agent DTT after co-immunoprecipitation resulted in the same band pattern as the one observed in lane 2, indicating that receptor dimerization and oligomerization that occurs in vivo is not sensitive to reducing agents and thus is not dependent on the formation of disulfide bonds.

\section{OTR oligomers are present at the cell surface and are modulated by ligand addition}

The experiments described above provided evidence for the existence of OTR oligomers; however, they did not provide any information with respect to the subcellular distribution of these oligomers. To answer specifically the question whether OTR oligomers were present at the cell surface, the antibody used for coimmunoprecipitation was added directly to intact living cells, thus limiting antibody access to cell surface molecules, as described (Zeng \& Wess 1999). To this end, cells expressing Myc-OTR as well as an OTR endowed with a triple N-terminal Flag tag $(3 \times$ Flag-OTR $)$ were exposed overnight to an anti-Flag antibody and, following extensive washing, antibody-tagged complexes were immunoprecipitated using protein $\mathrm{G}$ Sepharose beads and analyzed by immunoblotting using an anti-Myc antibody. As shown in Fig. 4, immune complexes containing Myc immunoreactivity could be efficiently precipitated by this strategy, indicating the presence of $3 \times$ Flag-OTR/Myc-OTR dimers or oligomers at the cell surface.

The role of receptor ligands in the formation of receptor oligomers is controversial. For some receptor systems, oligomerization is reported to be induced by agonists (Patel et al. 2002), suppressed by agonists (Gvejic \& Devi 1997) or independent of ligand addition (Ayoub et al. 2002). Therefore we investigated the role of OT addition on the amount of cell surface oligomers detectable with the present approach. The fact that, as described in the previous experiments, OTR oligomers were detected independently of ligand addition indicates that OTR oligomerization can occur in the absence 
of agonist. However, as shown in Fig. 4C, addition of agonist led to a rapid and significant decrease in the amount of detectable surface oligomer complexes recovered by co-immunoprecipitation.

\section{Evidence for OTR oligomerization in vivo using BRET}

The co-immunoprecipitation experiments described so far were based on the detection of receptor oligomers in cell extracts. Thus they provided strong evidence for, but no direct proof of, receptor oligomerization occurring in vivo. Therefore, we next investigated the existence of receptor oligomers in real time within living cells using BRET. Briefly, BRET is a technique used to detect protein/protein interaction in intact cells and is based on a non-radiative transfer of energy between the luminescent donor Renilla luciferase and the fluorescent acceptor YFP (Xu et al. 1999). Whereas light emission by luciferase is induced by substrate addition, the characteristic fluorescence emission of YFP is strictly dependent on its close proximity to luciferase upon luminescence emission. This technique has recently been used to assess oligomerization of GPCRs in intact cells (Angers et al. 2000, Eidne 2002). We assessed OTR oligomerization by BRET by transiently expressing in COS-7 cells two versions of the OTR carrying at their C-terminus either the Renilla luciferase protein (OTR-luc) or the YFP (OTR-YFP). Cells were exposed to the membrane-permeable Renilla luciferase substrate coelenterazine $\mathrm{h}$, and bioluminescence was measured using a spectrofluorometer. As shown in Fig. 5, co-transfection of cells with a luciferase-containing OTR-luc and OTR-YFP led to a marked and reproducible increase in the BRET ratio compared with OTR-luc-only expressing cells (bar 3 vs 1). Furthermore, no signal was detected when OTR-YFP was expressed alone, excluding any luciferase-independent fluorescence emission by YFP (bar 2). Three additional control experiments further validated these observations. First, no significant increase in the BRET ratio was observed when OTR-luc was co-expressed with free YFP, not attached to the OTR (bar 4). Secondly, no change in the BRET ratio relative to control was observed when a mix of two cell populations was used, each expressing separately OTR-luc and OTR-YFP (bar 5), demonstrating that both tagged receptors have to be co-expressed in the same cell in order to induce a change in the BRET ratio. Thirdly, no significant change in the BRET ratio was observed when the OTR-YFP plasmid was replaced by B2R-YFP, a plasmid encoding the human bradykinin $\mathrm{B} 2$ receptor fused to YFP (bar 6).

Finally we assessed the effect of agonist addition on the observed BRET ratio. In contrast to our findings using the co-immunoprecipitation approach, no significant change in the BRET ratio was observed upon the addition of agonist (bars 7 vs 8). However, the fact that a significant effect on the BRET ratio was observed in the absence of agonist is consistent with the previous observation that OTR oligomers were found in the absence of ligand.

\section{Discussion}

The present report provides evidence using two independent approaches that the OTR exists in dimeric and oligomeric forms at the cell surface in vivo. First, using a co-immunoprecipitation approach, we demonstrated the presence of SDS-sensitive and -resistant dimers and oligomers. By the use of sulfhydryl alkylating agents, we excluded the possibility that the observed co-immunoprecipitation was due to artefactual aggregation during receptor solubilization or immunoprecipitation. Moreover, OTR oligomerization does not involve the formation of disulfide bonds. Secondly, the concept of OTR oligomerization is further supported by the detection of BRET in intact living cells. Oligomerization was observed at receptor densities that were well within the physiological range.

The constitutive nature of OTR oligomers suggests that oligomerization may be involved in receptor folding and trafficking (Angers et al. 2002). Using a cell surface co-immunoprecipitation strategy, we determined that OTR oligomers are indeed present at the cell surface. Treatment of cells with OT led to a decrease of surface OTR recovered by co-immunoprecipitation, implying a change in receptor/receptor interactions. Because this phenomenon correlates, in time, with receptor activation, it is possible that it may be part of the receptor activation process. A transition of the receptor from an oligomeric to a monomeric state was recently proposed to be involved in the 


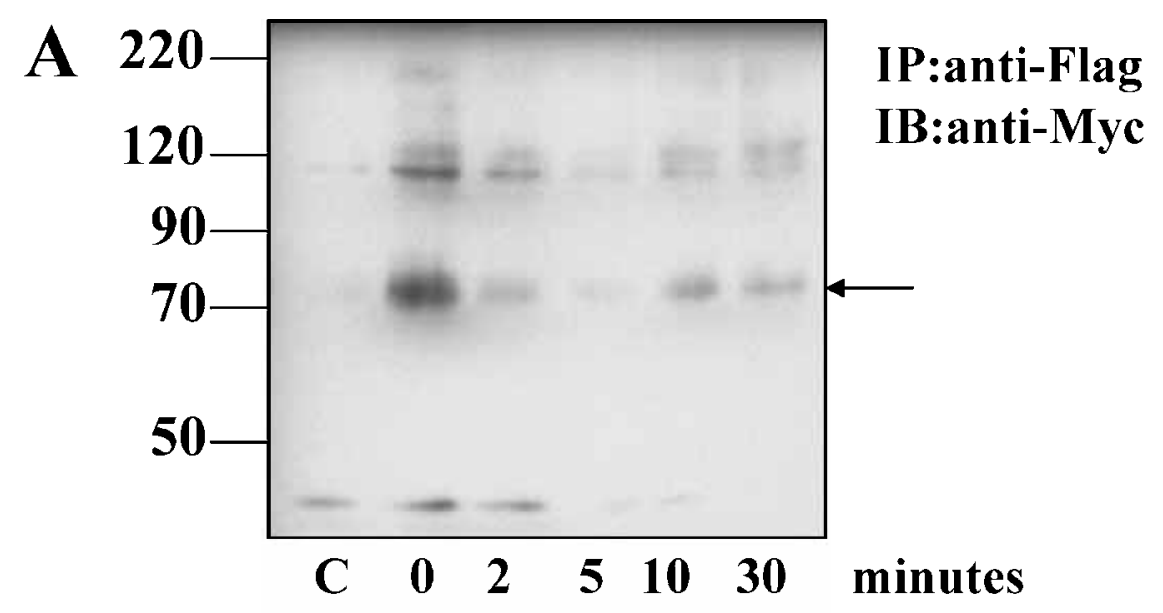

B
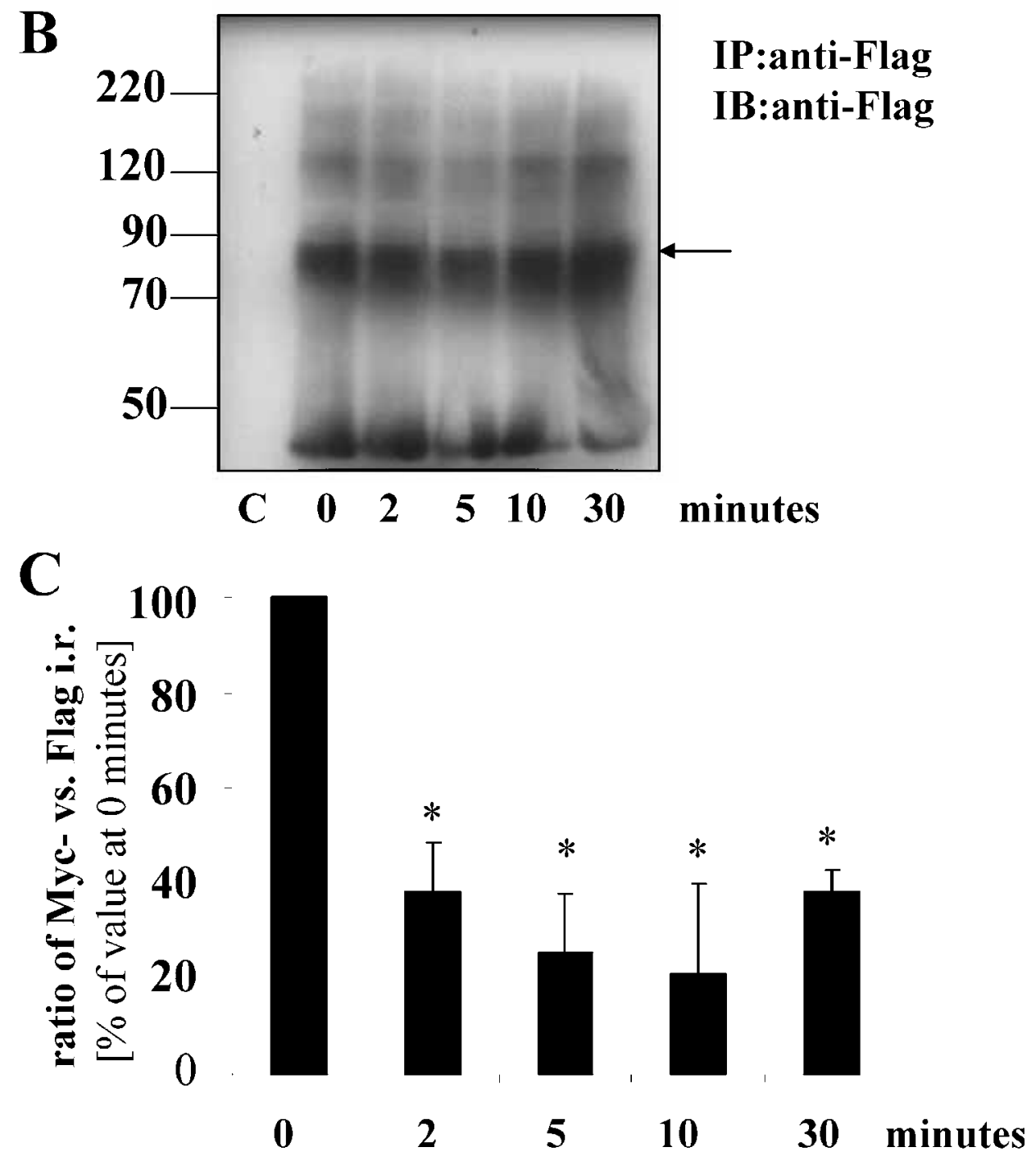


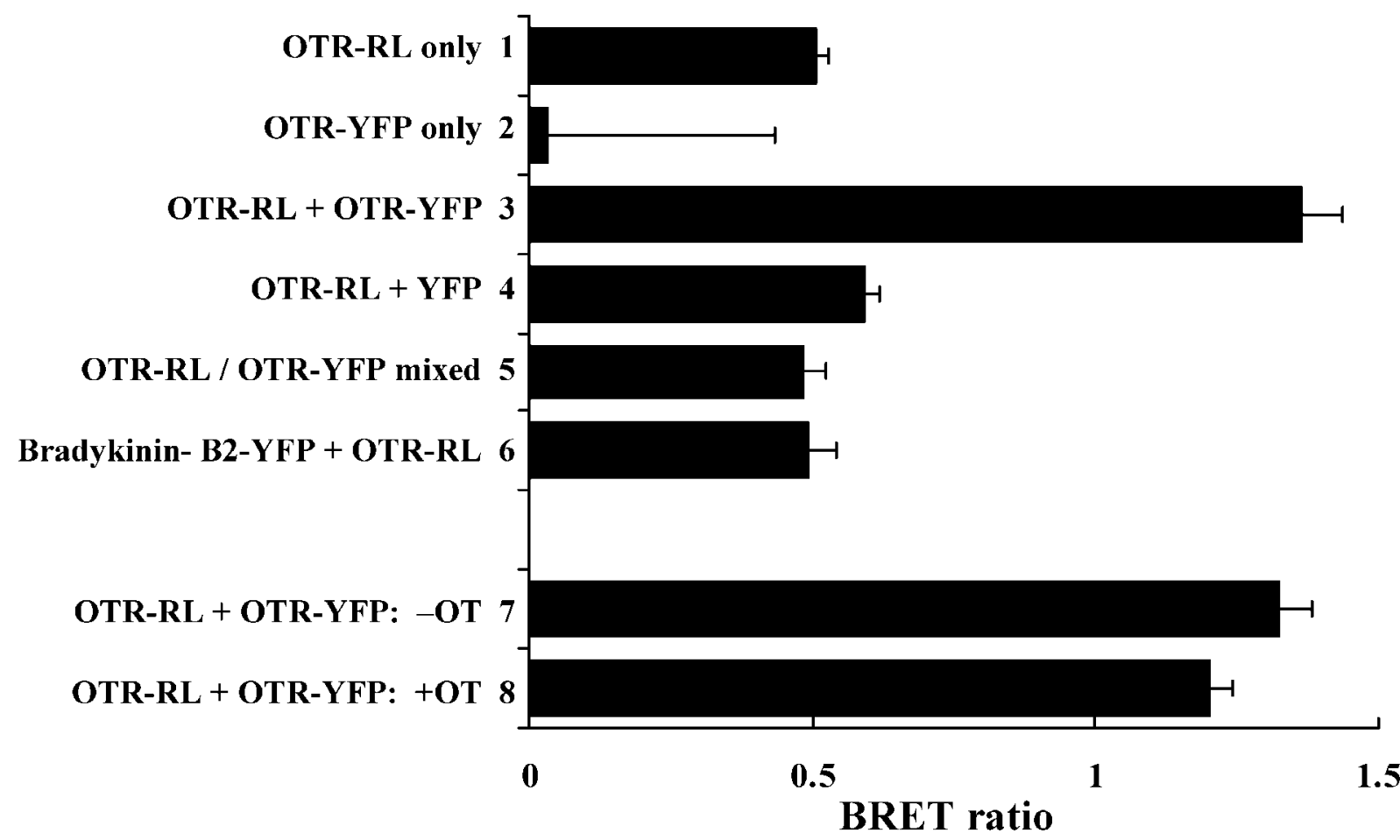

Figure 5 OTR oligomerization in living cells detected by BRET. COS-7 cells were transfected with either OTR-Iuc or OTR-YFP alone (lanes 1 and 2) or with both vectors in combination (lanes 3, 7 and 8). As a control, BRET was also assessed in cells co-transfected with OTR-luc and an expression vector for the YFP protein alone (lane 4) or in a mixture of two cell populations transfected separately with either OTR-luc or OTR-YFP (lane 5) and in cells co-transfected with OTR-luc and B2R-YFP, an expression vector encoding the human bradykinin B2 receptor fused to YFP (lane 6). To test the possible influence of agonist on the BRET ratio, cells were pretreated with $100 \mathrm{nM}$ OT or vehicle for $5 \mathrm{~min}$ at $37^{\circ} \mathrm{C}$ prior to BRET analysis (lanes 7 and 8). Each bar represents the mean \pm S.E.M. of BRET ratios determined in four independent experiments. Receptor densities were 425 and $310 \mathrm{fmol} / \mathrm{mg}$ protein for the OTR-YFP and B2R-YFP respectively.

activation of the thyrotropin receptor (Latif et al. 2002).

However, the possibility cannot be ruled out that agonist binding does not induce monomerization per se but induces a conformational change in the receptor structure that leads to a decrease in the stability of receptor oligomers and, consequently, to receptor monomerization during the immunoprecipitation step. This hypothesis could explain why, after agonist addition, we observed an apparent decrease in OTR cell surface oligomers with the co-immunoprecipitation approach but were unable to find any significant changes in the BRET ratio.

Figure 4 OTR oligomers are present at the cell surface and modulated by agonist exposure. (A) COS-7 cells transiently transfected with Myc-OTR and $3 \times$ Flag-OTR were treated at $37^{\circ} \mathrm{C}$ with $100 \mathrm{nM}$ OT for different times (as indicated) and incubated overnight at $4{ }^{\circ} \mathrm{C}$ with an anti-Flag antibody. As a control, cells transfected with Myc-OTR alone were also used (lane C). Immunocomplexes were isolated using protein G Sepharose. Co-immunoprecipitated Myc-OTR was visualized by immunoblotting with an anti-Myc antibody. Molecular mass markers (on the left) are in $\mathrm{kDa}$. (B) As a control for the efficiency of the immunoprecipitation reaction, the blot shown in (A) was immunoblotted using an anti-Flag antibody. (C) Quantitative analysis of Myc-OTR recovered by co-immunoprecipitation at different times after ligand addition. Bands indicated by arrows in ( $A$ and $B$ ) were quantitated densitometrically and the ratio of Myc immunoreactivity (as shown in A) vs Flag immunoreactivity (as shown in B) was plotted. Each bar represents the mean \pm S.E.M. of three independent experiments. Values are expressed as a percentage of the value at time 0. ${ }^{\star} P<0.05$ vs control as determined by ANOVA. IB, immunoblot; IP, immunoprecipitate. 
Among the members of the OT/vasopressin family, only the vasopressin V2 receptor has so far been shown to form oligomers. Both wild-type and a C-terminally truncated V2 receptor mutant can form SDS-resistant dimers (Hebert et al. 1996) and the functionality of inactive C-terminally truncated V2 receptor mutants can be rescued by $\mathrm{co}^{-}$ expression of a C-terminal receptor fragment (Schoneberg et al. 1996, 1997). On the other hand, certain N-terminally truncated mutants can act as dominant negatives and prevent the wild-type receptor from reaching the cell surface (Zhu \& Wess 1998). To what extent V2 receptor oligomers are present at the cell surface has remained controversial, however. Sadeghi et al. (1998) reported that $\mathrm{V} 2$ oligomerization was restricted to immature receptors present in the endoplasmic reticulum and that the function of the wild-type receptor remained unaltered, when co-expressed with a mutant V2 receptor lacking G-proteincoupling activity.

In the present study, we provide evidence that the OTR, another member of the OT/vasopressin family of receptors, forms oligomers that exist at the cell surface. Based on this finding, it is tempting to speculate that the OTR may form, in addition to homodimers, also heterodimers with other members of the GPCR family, including other members of the vasopressin receptor family. It remains to be determined to what extent the formation of heterodimers between the OTR and other members of the GPCR family may account for OT binding sites with altered pharmacological characteristics, such as the ones reported in the uterus (Chen et al. 1994).

The question remains, however, whether OTR oligomers and possible heterodimers are functional and what role, if any, they may play in the signaling process. The present observation of ligand-induced changes in the oligomerization status may indicate that, at least at the cell surface, the oligomerization process may be dynamic and thus represent an additional level of regulation of ligand-induced signaling processes.

\section{Acknowledgements}

We thank B Chini and S A Laporte for their gift of plasmids and C Russo for technical assistance. D Devost was supported by a fellowship from the
Research Institute of the McGill University Health Centre, Montreal, Canada. H H Zingg holds the first Wyeth-Ayerst, Canada Inc. Clinical Research Chair in Women's Health and a Senior Scientist Award from the Canadian Institutes for Health Research (GIHR). The research was supported by grants from CIHR and Wyeth-Ayerst, Canada Inc.

\section{NOTE ADDED IN PROOF}

While the present paper was under review, the formation of OTR dimers during biosynthesis was described by $\mathrm{S}$ Terrillon et al. (Terrillon S, Durroux T, Mouillac B, Breit A, Ayoub MA, Taulan M, Jockers R, Barberis C \& Bouvier M 2003 Oxytocin and vasopressin V1a and V2 receptors form constitute homo- and heterodimers during biosynthesis. Molecular Endocrinology 17 677-691).

\section{References}

Angers S, Salahpour A, Joly E, Hilairet S, Chelsky D, Dennis M \& Bouvier M 2000 Detection of beta 2-adrenergic receptor dimerization in living cells using bioluminescence resonance energy transfer (BRET). PNAS 97 3684-3689.

Angers S, Salahpour A \& Bouvier M 2002 Dimerization: an emerging concept for $\mathrm{G}$ protein-coupled receptor ontogeny and function. Annual Review of Pharmacology and Toxicology 42 409-435.

Ayoub MA, Couturier C, Lucas-Meunier E, Angers S, Fossier P, Bouvier M \& Jockers R 2002 Monitoring of ligand-independent dimerization and ligand-induced conformational changes of melatonin receptors in living cells by bioluminescence resonance energy transfer. Fournal of Biological Chemistry 277 21522-21528.

Breton C, Chellil H, Kabbaj-Benmansour M, Carnazzi E, Seyer R, Phalipou S, Morin D, Durroux T, Zingg H, Barberis C et al. 2001 Direct identification of human oxytocin receptor-binding domains using a photoactivatable cyclic peptide antagonist: comparison with the human Vla vasopressin receptor. Fournal of Biological Chemistry 276 26931-26941.

Chen DC, Chan WY \& Manning M 1994 Agonist and antagonist specificities of decidual prostaglandin-releasing and myometrial uterotonic oxytocin receptors in pregnant rats. Fournal of Reproduction and Fertility 102 337-343.

Cvejic S \& Devi LA 1997 Dimerization of the delta opioid receptor: implication for a role in receptor internalization. Fournal of Biological Chemistry 272 26959-26964.

Eidne KA 2002 Application of novel resonance energy transfer techniques to study dynamic hormone receptor interactions in living cells. Trends in Endocrinology and Metabolism 13 415-421.

Fanelli F, Barbier P, Zanchetta D, de Benedetti PG \& Chini B 1999 Activation mechanism of human oxytocin receptor: a combined study of experimental and computer-simulated mutagenesis. Molecular Pharmacology 56 214-225.

Fuchs A-R, Helmer H, Behrens O, Liu H-C, Antonian L, Chang S \& Fields M 1992 Oxytocin and bovine parturition: a steep rise in endometrial oxytocin receptors precedes onset of labor. Biology of Reproduction 47 937-944. 
Hawtin SR, Howard HC \& Wheatley M 2001 Identification of an extracellular segment of the oxytocin receptor providing. agonist-specific binding epitopes. Biochemical fournal 354 465-472.

Hebert TE, Moffett S, Morello J-P, Loisel TP, Bichet DG, Barret C \& Bouvier M 1996 A peptide derived from a beta 2-adrenergic receptor transmembrane domain inhibits both receptor dimerization and activation. Journal of Biological Chemistry 271 16384-16392.

Issafras H, Angers S, Bulenger S, Blanpain C, Parmentier M, Labbe-Jullie C, Bouvier M \& Marullo S 2002 Constitutive agonist-independent CCR5 oligomerization and antibody-mediated clustering occurring at physiological levels of receptors. Journal of Biological Chemistry 277 34666-34673.

Ku CY, Qian A, Wen Y, Anwer K \& Sanborn BM 1995 Oxytocin stimulates myometrial guanosine triphosphatase and phospholipase-C activities via coupling to $\mathrm{G}$ alpha $\mathrm{q} / 11$. Endocrinology 136 1509-1515.

Latif R, Graves P \& Davies TF 2002 Ligand-dependent inhibition of oligomerization at the human thyrotropin receptor. Fournal of Biological Chemistry 277 45059-45067.

Patel RC, Kumar U, Lamb DC, Eid JS, Rocheville M, Grant M, Rani A, Hazlett T, Patel SC, Gratton E et al. 2002 Ligand binding to somatostatin receptors induces receptor-specific oligomer formation in live cells. PNAS 99 3294-3299.

Postina R, Kojro E \& Fahrenholz F 1996 Separate agonist and peptide antagonist binding sites of the oxytocin receptor defined by their transfer into the V2 vasopressin receptor. Fournal of Biological Chemistry 271 31593-31601.

Sadeghi HM, Innamorati G, Esqueda E \& Birnbaumer M 1998 Processing and ligand-induced modifications of the V2 vasopressin receptor. Advances in Experimental Medicine and Biology 449 339-346.

Salahpour A, Angers S \& Bouvier M 2000 Functional significance of oligomerization of G-protein-coupled receptors. Trends in Endocrinology and Metabolism 11 163-168.
Schneider C, Newman RA, Sutherland DR, Asser U \& Greaves MF 1982 A one-step purification of membrane proteins using a high efficiency immunomatrix. Fournal of Biological Chemistry 257 10766-10769.

Schoneberg T, Yun J, Wenkert D \& Wess J 1996 Functional rescue of mutant $\mathrm{V} 2$ vasopressin receptors causing nephrogenic diabetes insipidus by a co-expressed receptor polypeptide. EMBO fournal 15 1283-1291.

Schoneberg T, Sandig V, Wess J, Gudermann T \& Schultz G 1997 Reconstitution of mutant V2 vasopressin receptors by adenovirus-mediated gene transfer. Molecular basis and clinical implication. Fournal of Clinical Investigation 100 1547-1556.

Strakova Z \& Soloff MS 1997 Coupling of oxytocin receptor to G proteins in rat myometrium during labor: Gi receptor interaction. American Fournal of Physiology 272 E870-E876.

Terrillon S, Durroux T, Mouillac B, Breit A, Ayoub MA, Taulan M, Jockers R, Barberis C \& Bouvier M 2003 Oxytocin and vasopressin V1a and V2 receptors form constitute homo- and heterodimers during biosynthesis. Molecular Endocrinology 17 677-691.

Xu Y, Piston DW \& Johnson CH 1999 A bioluminescence resonance energy transfer (BRET) system: application to interacting circadian clock proteins. PNAS 96 151-156.

Zeng FY \& Wess J 1999 Identification and molecular characterization of M3 muscarinic receptor dimers. Fournal of Biological Chemistry 274 19487-19497.

Zhu X \& Wess J 1998 Truncated V2 vasopressin receptors as negative regulators of wild-type V2 receptor function. Biochemistry 37 15773-15784.

Zingg HH \& Laporte SA 2003 The oxytocin receptor. Trends in Endocrinology and Metabolism $14222-227$.

Received in final form 9 June 2003

Accepted 24 July 2003

www.endocrinology.org 